\title{
McCune-Albright Syndrome-Presenting with Precocious Puberty
}

\author{
SETARA BINTE KASEM ${ }^{1}$, SHAIKH ABDUR RAZZAQUE ${ }^{2}$, RAISAADIBA ${ }^{3}$, SELMAANIKA ${ }^{4}$
}

\section{Introduction}

McCune-Albright Syndrome (MAS) is characterized by a triad of physical signs-café-au-lait pigmented skin lesions, polystotic fibrous dysplasia (FD) \& endocrine dysfunction, which particularly manifests as peripheral precocious puberty in girls. ${ }^{1,2}$ It is a rare disease with an estimated prevalence between $1 / 100,000$ and $1 / 1,000,000$. Thecafé-au-lait skin pigmentation consists of large hypermelanotic maculae of irregular and serpiginous (coast of Maine) borders which occur mainly on the front, posterior area of neck, buttocks, thorax, back, shoulder and pelvis. ${ }^{3}$ FD is a benign condition in which the medullary portion of the bone is replaced by poorly organized fibrous tissue with trabeculae of immature bone. It may affect either a single bone (monostotic) or numerous bones (polyostotic). It is caused by embryonic somatic mutation, leading to the substitution of His or Cys for Arg at aminoacid 201 of the Alpha-Subunit of the signal transduction protein Gs (Gs alpha). ${ }^{3}$ Recent studies have shown a clonal origin for FD, suggesting that this lesion is neoplastic in nature. The endocrine disorders associated with this disease may include precocious puberty, hyperthyroidism, pituitary adenomas, adrenal primary hyperplasia, hypophosphatemia and ovarian cysts. ${ }^{3}$ Sexual precocity is pubertal development occurring before the age of 8 years in girls, and before the age of 9 years in boys. ${ }^{4}$ In MASprecocious puberty is the common clinical presentation and girls are affected more than boys. ${ }^{3}$ Affected children usually present at a younger age than those with idiopathic precocious puberty. ${ }^{4}$ The non classical form of MAS consists of only two of these three conditions. ${ }^{3}$ Here we report a case of MAS with this triad of symptoms.

1. Assistant Professor (Gynae \& obstetrics), Sir Salimullah Medical College and Mitford Hospital

2. Associate Professor (Paediatric Cardiology), National institute of Cardiovascular diseases.

3. $5^{\text {th }}$ year student, Holly Family Red Cresent Hospital.

4. $1^{\text {th }}$ year student, Bangladesh Medical College

Correspondence: Dr. Setara Binte Kasem. Assistant Professor, House no-40, Flat-B2, Road-10A, Dhanmondi, Dhaka, E maildr.setaraa @ gmail.com. Mob-01552314895

\section{Case Report}

Our patient was referred to department of Gynaecology and Obstetrics from department of Paediatric surgery with the complaints of per vaginal $(\mathrm{P} / \mathrm{V})$ bleeding and ovarian cyst (ultrasonographic finding) at her two and half years of age. Her guardian stated that she presents with multiple café-au-lait macules all over the body since birth. At her 16 months of age she developed per vaginal bleeding which was scanty in amount and persisted for three days. Then she continued scanty P/V bleeding 2-3 monthly (persisted for 2-3 days). They noticed slight enlargement of her breasts. They consulted witha local doctor of Belabo,Norshindi but was not cured.At her one and half years of age she developed bony deformities, pain in extremities and limping gait. Due to fall on a ground at her two and half years of age her right forearm was fractured.

On examination- Patient's height was $89 \mathrm{~cm}$, weight was $11 \mathrm{~kg}$, her temperature was $98.4^{\circ} \mathrm{F}$, Pulse-80/ min,BP-90/55 mm of $\mathrm{Hg}$, Her lymph nodes and thyroid gland were not enlarged, Heart and chest were normal. Her breast buds were developed as tanner stage 2-3 (Fig.-1) without galactorrhoea.Multiple skin pigmentation were present on all over the body eg: back of the neck, back of the chest, back of the buttock, back of the thigh, front of the arm and front of

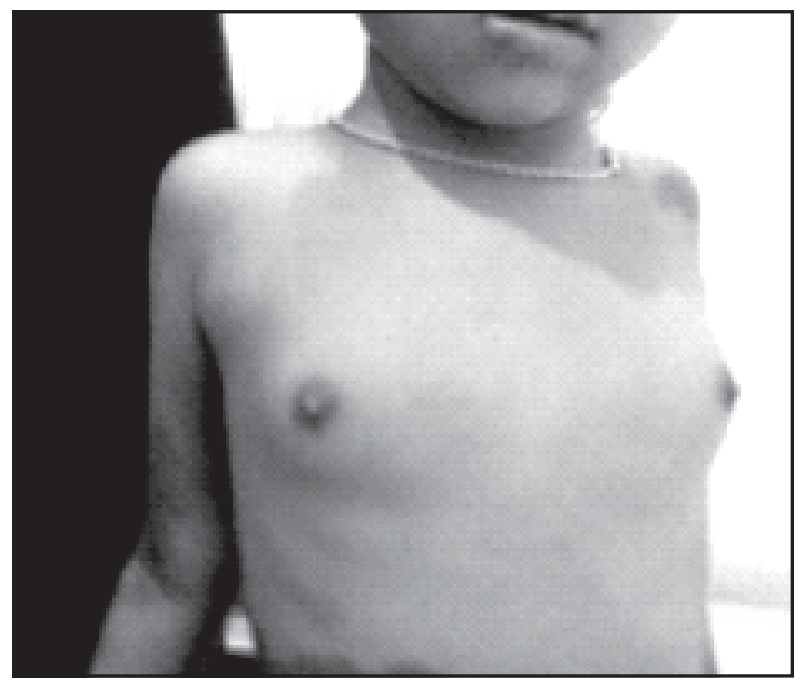

Fig.-1: Breast enlargement due to prtecocious puberty 
the abdomen (Fig-2,3,4). There was fixed bending of right thumb and slight deformities of lower limbs. She had no axillary or pubic hair growth. On per abdominal examination-no tenderness or palpable mass was present. Per vaginal examination revealed clitoris and labia minora were well developed \& introitus was intact.

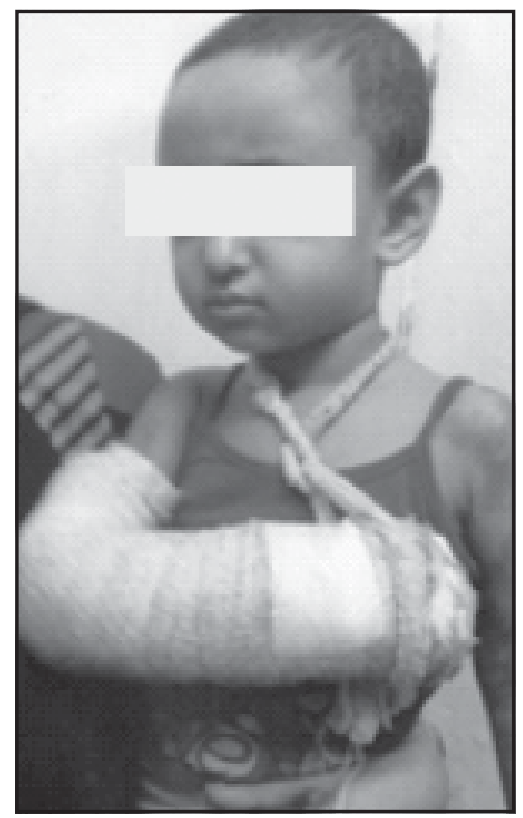

Fig.-2: Fracture of right forearm

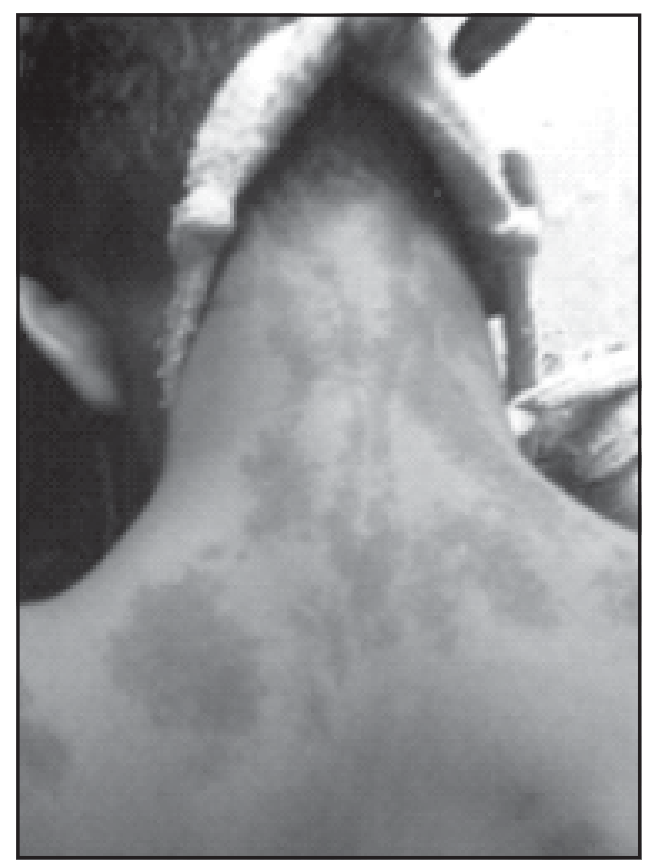

Fig.-3: Café-aulait pigmentain

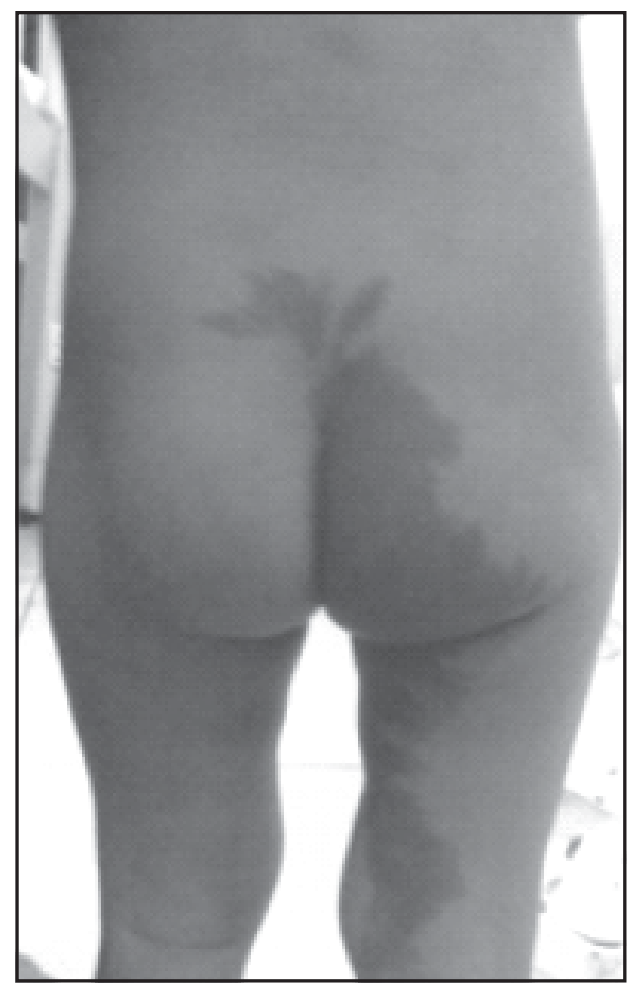

Fig.-4: Café-au-lait pigmentation \& limb deformities

Laboratory data was as follows : Hb\%-12.5gm/ $\mathrm{dl}, \mathrm{WBC}-7000 / \mathrm{mm}^{3}$, RBS- $5.3 \mathrm{mmol} / \mathrm{L}$,Serum creatinine-.8mg/dl (0.67-1.2), SGPT- 35U/L (upto 40), CA 125-42.9 U//ml(normal<35.00), carcinoembryonic antigen(CEA)-1.0ng/ml, Serum calcium-8.6mg/dl (8.410.2), Serum phosphorus(inorganic)-4.5mg/dl(2.54.9).Endocrinological evaluation revealed:an elevated level of estradiol $34.06 \mathrm{pg} / \mathrm{ml}$ (prepubertal undetectable to $20 \mathrm{pg} / \mathrm{ml}$ ), FSH-1.26 IU/L (Prepubertal<1.16 IU/L), LH-1.50 IU/L(Prepubertal 0.56-1.91IU/L), Testosteron$8.24 \mathrm{ng} / \mathrm{dl}$ (females 63-120 ng/ml), Prolactin- $407 \mathrm{mlU} /$ L(Female <460mIU/L), TSH-0.36mIU/L (Children0.366.0), $\mathrm{FT}_{4}-13.39 \mathrm{pmol} / \mathrm{l}$ (Normal 9.14-23.18), $\mathrm{T}_{3}$ 2.59nmol/L (Normal 1.21-3.08) ACTH-9.85pg/ml(ND to 46), Cortisol-192.5nmol/L (Normal 138-690 nmol/L).

Pelvic ultrasound showed uterus was bigger in size $(4.4 \times 1.6 \mathrm{~cm})$ with respect to her age \& there were bilateral follicular ovarian cysts (right one was about $2.9 \times 2 \mathrm{~cm}$ and left one was about $1.9 \times 1.2 \mathrm{~cm}$ ). No collection was seen in pouch of Douglas (Fig.-5). Ultrasonogram of both breasts showed echotexture of both breasts parenchyma were prominent with respect to her age. 


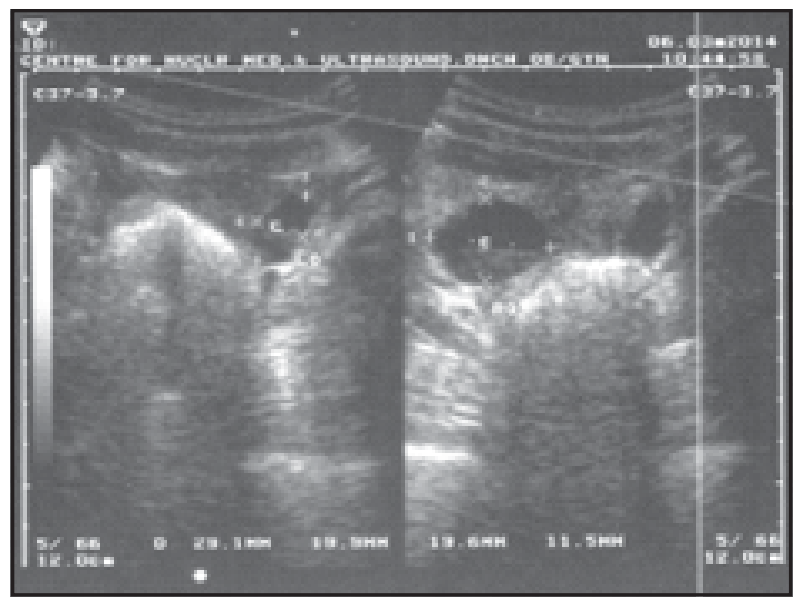

Fig.-5: Bilateral ovarian cysts

MRI of Brain with contrast showed features suggestive of extensive fibrous dysplasia involving base of the skull including clivus and right maxilla. No evidence of intracranial haemorrhage, territorial infarct or intracranial mass lesion. $\mathrm{X}$ ray of arm and forearm showed green stick fracture of distal shaft of right radius and ulna with generalized osteopenia. $X$ ray of hand and foot showed widened spongy bones of the hand and foot with coarse trabeculation and multiple small cystic areas. There were fibrodysplastic changes and thinning of the cortical bones of the hand and foot (Fig.-6).X ray wrist joint showed inner epiphysis of humerus already appeared, which corresponds with

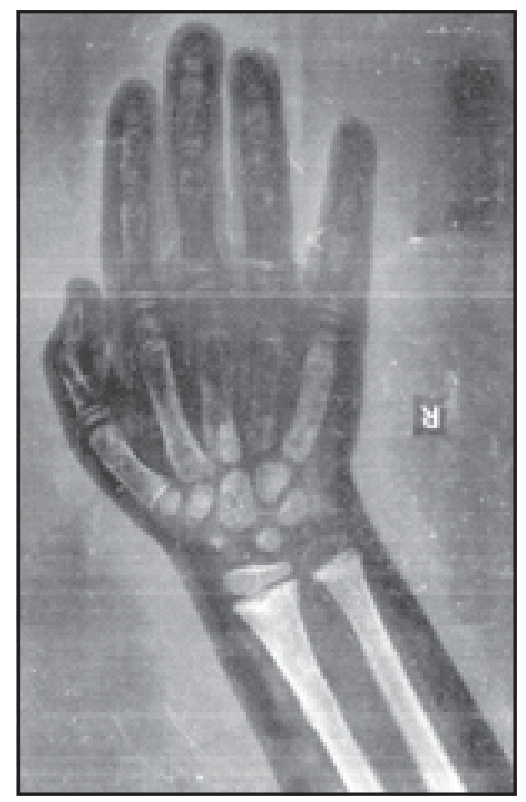

Fig.-6: X-ray of hand and wrist joint fibrodysplastic changes, bending right thumb age $>5$ years. Again seven carpal bones have appeared, Pisiform bone was not appeared, which corresponds age $<7$ years. So, bone age corresponds age range between 5 to 7 years(Fig.-6).

All these results were consistent with diagnosis of McCune-Albright syndrome with precocious puberty. Initially patient was treated with Inj. GnRH analogue (Depot leuprolide acetate 300-500ìg/kg) monthly for precocious puberty. Plaster and immobilization was applied for fracture. Her P/V bleeding had stopped one month after starting of Inj. GnRH analogue and she was cured from fracture after three months. There was no further growth of her breasts. On USG no ovarian cyst was found after six months. Bisphosphonate therapy had started $(0.4 \mathrm{mg} / \mathrm{kg}$ body weight every four weeks) for FD. Her limb pain had remitted significantly.

\section{Discussion}

MAS is a rare multisystem disorder, first described in 1937 separately by Donovan McCune and Fuller Albright in a group of children, mostly females, with skin pigmentation, bone deformities and endocrine disorders that develops from an activating mutation in the Gs gene. The disorder is the result of post zygotic somatic mutation in the gene GNAS1 on chromosome 20q13-13.29, coding for the á subunit of stimulatory G protein. ${ }^{3}$ One of the main characteristics of these endocrinopathies is that they are autonomous: the metabolic disorders are not accompanied by elevated plasma concentration of the stimulating pituitary hormone or hypothalamic releasing factor. The endocrine glands that are hyperactive in MAS also have in common a response to extracellular signals by the adenylate cyclase(AC) cAMP pathway. ${ }^{5}$

The clinical expression depends on the number of mutated cells and affected organs. Thus the presentation can be heterogenous, involving various endocrine and non endocrine organs. It can be early or late onset or slow or quick evolution. This abnormality lead to proliferation of osteoprogenitor cells without differentiation, therefore fibrous matrix with woven bone increase.MAS is diagnosed on the triad of FD, endocrinopathy and hyperpigmentation of the skin. ${ }^{3}$ The clinical presentation of MAS is highly variable, depending on which of the various potential components of the syndrome 
predominate. The classical form of MAS is more common in females and is defined by physical signs as descrbed above ${ }^{3}$.

Café-au- lait macules (CALMs) develop between the age of 4 months and 2 years, but may be present at birth. ${ }^{3} \mathrm{FD}$ usually presents with bone pain, deformities, recurrent pathological fracture of the affected site. ${ }^{5}$ Abnormal fibrous tissue growth occurs in many bones, especially in the long bones, ribs and skull bones. FD ranges fron small asymptomatic areas, detected only by bone scan, to markedly disfiguring lesions, resulting in pathological fracture and impringement on vital structures. ${ }^{3}$

There is no specific treatment for this syndrome which is generally symptomatic. Because of the great variety of lesions in this syndrome, treatment is specific for each patient. Some data suggest that the cancer incidence in adulthood is increased in FD patient. ${ }^{6} \mathrm{To}$ date, prenatal diagnosis is not possible. But recently, through novel polymerase chain reaction-based techniques, activating mutation in the peripheral blood of patients with MAS has been successfully detected, which might help in diagnostic as well therapeutic areas. $^{3}$

Various medical and surgical treatment are offered for endocrine and non endocrine involvement according to signs and symptoms. Corrective surgery can be performed for bone dysplasia. Recently bisphosphonate therapy has shown promise for fibrous dysplasia patients as it helps to diminish pain, prevents fracture and leads to partial resolution of fibrous dysplasia lesions. ${ }^{3}$ Chemically, bisphosphonates, among which pamidronic acid is commonly used, are simple pyrophosphate derivatives interfering with phosphate metabolism in osteoclasts. This leads to inhibition or, ultimately, apoptosis of these cells. In children and adolescents, bisphosphonate therapy appears to be well tolerated in general, even in long term treatment. ${ }^{7}$ Therapy with bisphosphonates may reduce the co-morbidities. ${ }^{8}$ Long term( $>5$ years) bisphosphonates therapy is associated with severe suppression of bone turn over and results in atypical fracture of shaft of long bones. ${ }^{8}$

This paper reports a case of MAS in a two and half years old female of a lower middle socioeconomic rural community in whom diagnosis was delayed upto two and half years. Here Inj. GnRH analogue suppress the pituitary gonadotropin and halt the precocious puberty. The dosage can be adjusted upward or downward on the basis of clinical and laboratory findings. For precocious puberty aromatase inhibitors can use in girls with persistent estradiol elevation. Patient who respond to treatment should continue therapy until the age of normal puberty or until a bone age of 15-16 years.

\section{Conclusion}

The precocious puberty can also be an early manifestation of McCune-Albright syndrome and etiologic diagnosis of early sexual precocity is based on careful history and physical examination. Children with precocious puberty should be evaluated for endocrinopathies and hormonal study may be necessary in such cases. Prognosis for children with MAS is unfavourable. Adult height is significantly reduced. Multiple endocrinopathies often exist as well.

\section{References}

1. N Kalfa. P Philibert. F Audran. A Ecochard. T Hannon. S Lumbroso and C Sultan. Searching for somatic mutations in McCune-Albright syndrome: a comparative study of the peptidic nucleic acid versus the nested PCR method based on 148 DNA samples. European Journal of endocrinology. 2006;155: 839-43.

2. Albright F.Butler A. Hampton A and Smith P.Syndrome characterized by osteitis fibrosa disseminate, areas of pigmentation and endocrine dysfunction, with precocious puberty in females: report of five cases. New England Journal of Medicine. 1937; 216: 727-46.

3. Moein Mobini, Rahim Vacili, Saba Vakili. McCune-Albright Syndrome: A case report and literature Review. International Journal of Pediatrics. 2014 Apr; Vol 2,N.2-2, Serial No. 5: 153-156.

4. Alan H.Decherney, Lauren Nathan, Neri Laufer, Ashley S.Roman. Current Diagnosis and Treatment, Obstetrics and Gynecology. $11^{\text {th }}$ edition. 2013. p, 98.

5. Serge Lumbroso, Francoise Paris, and Charles Sultan. Activating Gsá Mutations: Analysis of 113 
Patients with Signs of McCune-Albright SyndromA European Collaborative study. The Journal of clinical Endocrinology \& Metabolism. 2004; 89(5): 2107-2113.

6. 5. Sepideh Siadati MD, Ensiyeh Shafigh MD. McCune-Albright Syndrome: A case report. Archives of Iranian Medicine. 2010; 13(3): 245-247.

7. Carl Friedrich Classen, Monika Mix, Ulrike Kyank, Christina Hauenstein and Dieter Haffner. Pamidronic acid and cabergoline as effective long term therapy in a 12-year-old girl with extended facial polyostotic fibrous dysplasia, prolactinoma, acromegaly in Mc Cune-Albright syndrome: a case report. Classen et al. Journal of medical case reports 2012, 6:32.

8. Sanjay Kumar Bhadada, Anil Bhansali, Sambit Das, Ramanbir Singh, R.Sen, A. Agarwal, BR.Mittal,Uma Nahar, Pinaki Dutta \& Niranjan Khandelwal. Fibrous dysplasia \& McCuneAlbright Syndrome: An experience from a tertiary care centre in north India. Indian J Med. May 2011; Res 133: 504-09. 